\title{
Homocysteine editing and growth inhibition in Escherichia coli
}

Correspondence
Hieronim Jakubowski
jakubows@umdnj.edu

Received 10 December 2008

Revised 2 February 2009

Accepted 11 March 2009

\author{
Marta Sikora ${ }^{1,2}$ and Hieronim Jakubowski ${ }^{1,2}$ \\ ${ }^{1}$ Department of Microbiology and Molecular Genetics, UMDNJ-New Jersey Medical School, \\ International Center for Public Health, Newark, NJ 07101, USA \\ ${ }^{2}$ Institute of Bioorganic Chemistry, Polish Academy of Sciences, Poznań, Poland
}

\begin{abstract}
In Escherichia coli homocysteine (Hcy) is metabolically converted to the thioester Hcy-thiolactone in ATP-consuming reactions catalysed by methionyl-, isoleucyl- and leucyl-tRNA synthetases. Here we show that growth inhibition caused by supplementation of $E$. coli cultures with Hcy is accompanied by greatly increased accumulation of Hcy-thiolactone. Energy dissipation for Hcy editing increases 100-fold in the presence of exogenous Hcy and reaches one mole of ATP unproductively dissipated for Hcy-thiolactone synthesis per each mole of ATP that is consumed for methionine activation. Inhibiting Hcy-thiolactone synthesis with isoleucine, leucine or methionine accelerates bacterial growth in Hcy-supplemented cultures. Growth rates in Hcyinhibited cultures are inversely related to the accumulation of Hcy-thiolactone. We also show that the levels of protein $N$-linked Hcy modestly increase in $E$. coli cells in Hcy-supplemented cultures. The results suggest that Hcy editing restrains bacterial growth.
\end{abstract}

\section{INTRODUCTION}

The non-protein amino acid homocysteine (Hcy) is an intermediate in the metabolism of the essential protein amino acid methionine. The accumulation of Hcy and its metabolite, the thioester Hcy-thiolactone, observed in genetic or nutritional deficiencies in Hcy metabolism (Chwatko et al., 2007), has also been implicated in cardiovascular and neurodegenerative disorders, including dementia and Alzheimer's disease (Jakubowski, 2001a, 2004, 2006a, 2007a). The formation of Hcy-thiolactone is a consequence of error editing reactions during protein biosynthesis (Jakubowski, 2005a, b). In all organisms, Hcy enters the first step of protein biosynthesis and forms an Hcy-AMP intermediate in a reaction catalysed by methionyl-tRNA synthetase (MetRS) (Jakubowski, 1990, 1991, 2001b). However, the misactivated Hcy is then edited, with the formation of the thioester Hcy-thiolactone (Jakubowski \& Fersht, 1981; Jakubowski, 1999a). In Escherichia coli two other enzymes, isoleucyl-tRNA synthetase (IleRS) and leucyl-tRNA synthetase (LeuRS), in addition to MetRS, participate in Hcy editing and Hcy-thiolactone biosynthesis (Jakubowski, 1995) (Fig. 1). As a result of these editing reactions, Hcy is not transferred to tRNA and is thus prevented from being translationally incorporated into protein (Jakubowski \& Goldman, 1992; Jakubowski, 2000, 2001b, 2005b) (Fig. 1).

Abbreviations: DTT, dithiothreitol; Hcy, homocysteine; MetRS, methionyltRNA synthetase; lleRS, isoleucyl-tRNA synthetase; LeuRS, leucyl-tRNA synthetase.
Excess Hcy is known to inhibit growth of microbial cells, such as the bacterium Escherichia coli (Karkhanis et al., 2007; Roe et al., 2002; Tuite et al., 2005) and the yeast Saccharomyces cerevisiae (Jakubowski, 1991; Kumar et al., 2006; Zimny et al., 2006), and to cause death of mammalian (Jakubowski, 2006a, 2007a) and yeast cells (Jakubowski, 2002a). The detrimental effects of Hcy in yeast and mammalian cells are linked to its conversion to Hcy-thiolactone, which can be harmful for two reasons. First, the conversion of Hcy to Hcy-thiolactone requires ATP and thus causes nonproductive dissipation of cellular energy (Fig. 1), which could inhibit growth, as demonstrated in yeast (Jakubowski, 1991). Second, the thioester Hcy-thiolactone is a reactive intermediate that causes protein modification ( $N$-homocysteinylation) through the formation of isopeptide bonds with $\varepsilon$-amino groups of protein lysine residues (Fig. 2) (Jakubowski, 1997a, 1999b), which alters or impairs the protein's function, ultimately leading to growth inhibition and cell death, a pathway well documented in mammalian and human cells (Jakubowski, 2006a, 2007a).

The aim of the present work was to determine whether any of these mechanisms contributes to the growth inhibition by Hcy in E. coli.

\section{METHODS}

Bacterial strains and growth conditions. Wild-type E. coli strain MG1655 and its protease-deficient variants, PhB1907 ( $\Delta c l p X P)$,

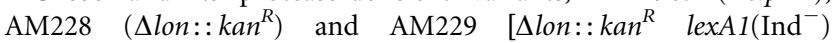
malF:: $\left.\operatorname{Tn} 10\left(\mathrm{Tet}^{\mathrm{R}}\right)\right]$ were kindly provided by Zafri Humayun's lab 


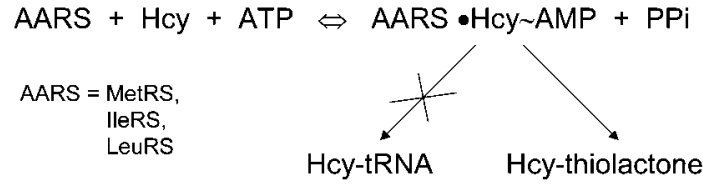

Fig. 1. Conversion of Hcy to Hcy-thiolactone.

(UMDNJ-New Jersey Medical School). Cells were grown in a citrate/ phosphate medium ( $\mathrm{pH} 6.0$ ) containing $0.2 \%$ glucose, $20 \mathrm{mM}$ citric acid, $56 \mathrm{mM} \mathrm{Na}_{2} \mathrm{HPO}_{4}, 5 \mathrm{mM} \mathrm{K} \mathrm{HPO}_{4}, 0.4 \mathrm{mM} \mathrm{MgSO}_{4} .7 \mathrm{H}_{2} \mathrm{O}$, $7.6 \mathrm{mM}\left(\mathrm{NH}_{4}\right)_{2} \mathrm{SO}_{4}, 4 \mu \mathrm{M}$ thiamine and $6 \mu \mathrm{M} \mathrm{FeSO}$. The medium was supplemented with DL-Hcy $(0.5-8 \mathrm{mM})$ and/or isoleucine $(0.2-$ $1.6 \mathrm{mM})$, leucine $(0.2-1.6 \mathrm{mM})$ or methionine $(0.2-1.6 \mathrm{mM})$ as indicated in the Results. All amino acids were from Sigma-Aldrich. Only the L-stereoisomer of Hcy is known to be edited by aminoacyltRNA synthetases (Jakubowski, 2005a). Fresh overnight cultures were diluted 10-20-fold into fresh medium. The cultures were maintained at $37{ }^{\circ} \mathrm{C}$ with shaking (200 r.p.m.) in $125 \mathrm{ml}$ side-arm flasks for $8 \mathrm{~h}$ and the growth was monitored using a Klett-Summerson colorimeter. Specific growth rates were calculated from the relationship $\mu=\ln 2 / t$, where $t$ is doubling time in hours. Representative results of two or three independent experiments are shown in Figs 3-6.

Determination of Hcy-thiolactone. At the end of each growth experiment aliquots of the E. coli cultures $(300 \mu \mathrm{l})$ were clarified by microcentrifugation $\left(14000 \mathrm{~g}, 2 \mathrm{~min}, 4{ }^{\circ} \mathrm{C}\right)$ and cell-free media were stored at $-20{ }^{\circ} \mathrm{C}$ until analyses. Cell-free media $(10 \mu \mathrm{l})$ from Hcysupplemented cultures were diluted 100-fold with water and $10 \mu \mathrm{l}$ of the diluted sample was applied to a cation-exchange HPLC column (Chwatko \& Jakubowski, 2005; Jakubowski, 2002a).

Determination of protein $\boldsymbol{N}$-linked Hcy. Protein $N$-linked Hcy was assayed as previously described (Jakubowski, 2002b, 2008) with the following modifications. E. coli cultures were grown to 100 Klett units $\left(2 \times 10^{8}\right.$ cells $\left.\mathrm{ml}^{-1}\right)$, and then supplemented with $4 \mathrm{mM}$ DL-Hcy. At timed intervals, cells from $5 \mathrm{ml}$ aliquots were collected by centrifugation and stored frozen at $-20{ }^{\circ} \mathrm{C}$ until analyses. Cells were resuspended in $0.5 \mathrm{ml} 50 \mathrm{mM}$ sodium phosphate buffer, $\mathrm{pH} 7.4$, $1 \mathrm{mM}$ DTT, $0.1 \mathrm{mM}$ EDTA and protein was extracted by sonication $(3 \times 15 \mathrm{~s}$, with cooling on ice). Crude extracts were clarified by centrifugation and protein concentration was determined by using a Bio-Rad reagent. Free Hcy was removed from the protein samples by eight cycles of filtration $(30 \mathrm{~min}, 10000 \mathrm{~g}$ ) and dilution (10-fold each) with the DTT-containing buffer by using a Millipore $10 \mathrm{kDa}$ molecular mass device. The DTT-washed protein samples were hydrolysed with $6 \mathrm{M} \mathrm{HCl}$ containing $0.1 \mathrm{M} \mathrm{DTT}$ at $120^{\circ} \mathrm{C}$ for $1 \mathrm{~h}$.

The hydrolysates were lyophilized, dissolved in $5 \mu \mathrm{l}$ water and $3 \mu \mathrm{l}$ aliquots subjected to two-dimensional thin-layer chromatography on $5 \times 4 \mathrm{~cm}$ cellulose plates (Analtech). DL- $\left.{ }^{35} \mathrm{~S}\right] \mathrm{Hcy}$-thiolactone (40 $000 \mathrm{Ci} \mathrm{mol}^{-1}, 7000$ c.p.m. per plate) (Jakubowski, 2000, 2007b) was added as a tracer. The plates were developed with butanol/acetic acid/water (4:1:1, by vol.) in the first dimension and with 2propanol/ethyl acetate/water/ammonia $(12: 12: 1: 0.12$, by vol.) in the second dimension. Hcy-thiolactone, localized by autoradiography using Kodak BioMax MR film ( $4 \mathrm{~h}$ exposure at $4{ }^{\circ} \mathrm{C}$ ), was eluted with $60 \mu \mathrm{l} 2 \mathrm{mM} \mathrm{HCl}$ and quantified by HPLC.

HPLC analyses. HPLC analyses were carried out using System Gold Nouveau HPLC instrumentation from Beckman-Coulter with a Jasco 1520 fluorescence detector and cation-exchange polysulfoethyl aspartamide column $(2 \times 35 \mathrm{~mm}, 5 \mu, 300 \AA)$ from PolyLC, as previously described (Chwatko \& Jakubowski, 2005; Jakubowski, 2002b, 2008).

\section{RESULTS AND DISCUSSION}

\section{Supplementation with Hcy greatly increases Hcy- thiolactone accumulation and causes growth inhibition in $E$. coli}

E. coli cells grown on minimal media produce Hcythiolactone in a reaction catalysed by MetRS (Jakubowski, 1990), in which Hcy reacts with ATP to form homocysteinyl adenylate (Hcy-AMP) which is then converted to Hcy-thiolactone and AMP (Jakubowski \& Fersht, 1981; Jakubowski, 1999a) (Fig. 1). Because its amino group has an exceptionally low $\mathrm{p} K_{\mathrm{a}}, 6.7$ (Jakubowski, 2006b), Hcythiolactone is mostly neutral under physiological conditions, diffuses out of the cells, and accumulates in the culture medium (Gao et al., 1994; Jakubowski, 1990, 1995). We found that in E. coli MG1655, PhB1907, AM228 and AM229 cultures grown on minimal medium for $8 \mathrm{~h}$, the levels of Hcy-thiolactone were 1.0, 5.0, 2.4, and $1.4 \mu \mathrm{M}$, respectively.

To determine whether metabolic conversion of Hcy to Hcy-thiolactone can affect growth rate, E. coli cultures were grown in minimal medium with $0.4 \%$ glucose as a carbon source supplemented with up to $8 \mathrm{mM}$ DL-Hcy, growth was monitored by using the Klett-Summerson apparatus, and the concentrations of Hcy-thiolactone in the medium were determined at the end of each growth experiment. We found that growth of E. coli strains MG1655, PhB1907, AM228 and AM229 was inhibited by DL-Hcy, with the inhibition increasing at higher DL-Hcy concentrations (Fig. 3a). We also found that Hcy-thiolactone concentrations in bacterial cultures increased up to 100-fold above basal levels (to 200-400 $\mu \mathrm{M}$ ) with increasing DL-Hcy concentration, with a higher Hcy-thiolactone concentration observed in PhB1907 and AM228 cultures than in the MG1655 culture (Fig. 3b). Exogenous Hcy-thiolactone added at

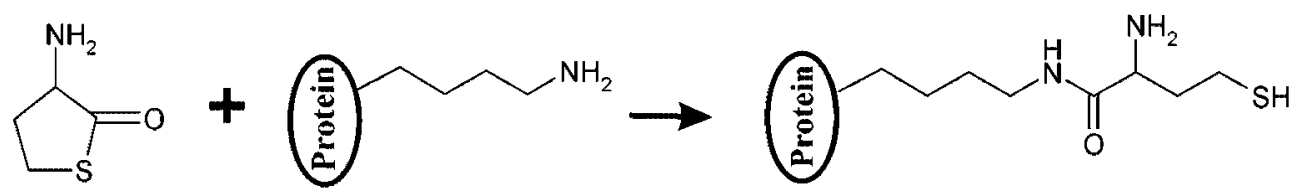

Fig. 2. Protein modification ( $N$-homocysteinylation) by Hcy-thiolactone. 

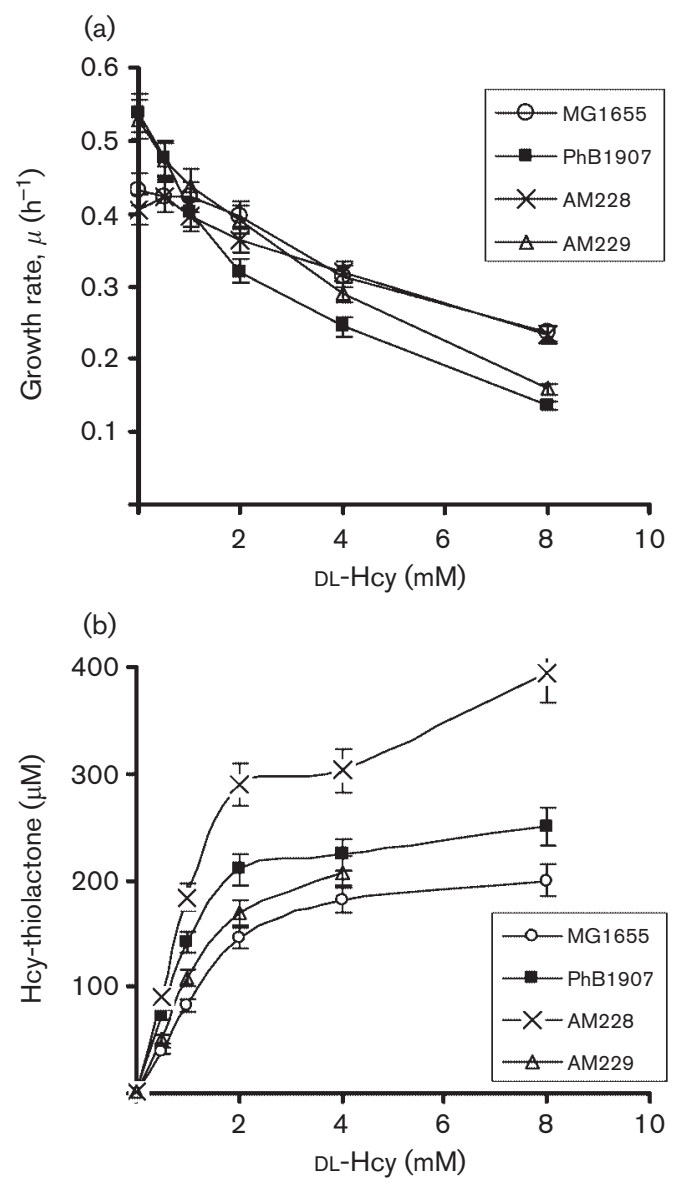

Fig. 3. Growth inhibition and increased Hcy thiolactone accumulation in E. coli cultures supplemented with DL-Hcy. E. coli strains MG1655, PhB1907, AM228 and AM229 were grown in minimal medium supplemented with the indicated DL-Hcy concentrations. Growth was monitored with a Klett-Summerson colorimeter. Hcy-thiolactone was assayed at the end of growth $(8 \mathrm{~h})$ as described in Methods. Growth rates (a) and Hcythiolactone concentrations (b) are plotted as a function of DLHcy concentration.

these concentrations to the growth medium does not inhibit growth of E. coli (Tuite et al., 2005).

\section{Acetate causes growth inhibition but does not affect Hcy-thiolactone accumulation in $E$. coli cultures}

It has been reported that growth inhibition of $E$. coli by sodium acetate is due to the accumulation of Hcy in the bacterial cells (Roe et al., 2002). Because the accumulation of Hcy leads to the accumulation of Hcy-thiolactone (Gao et al., 1994; Jakubowski, 1990, 1995, 2002a), we set out to determine whether the conversion of $\mathrm{Hcy}$ to Hcythiolactone plays a role in acetate-dependent growth inhibition. We measured growth rates and the levels of
Hcy-thiolactone in cultures of E. coli strains MG1655 and AM229 in minimal medium supplemented with a range of sodium acetate concentrations from 0 to $20 \mathrm{mM}$. We found that acetate inhibited the growth of our two E. coli strains, which is consistent with previous results obtained for E. coli strain Frag1 (a K-12 strain) (Roe et al., 2002). Unexpectedly, the supplementation with sodium acetate lowered (2- to 6-fold) the basal Hcy-thiolactone concentrations for the two bacterial strains examined (Fig. 4), which excludes the possibility that Hcy-thiolactone is involved in acetate-dependent growth inhibition. These results, in conjunction with our findings that Hcythiolactone accumulation increases up to 100 -fold over the basal levels with increasing Hcy concentrations (Fig. 3b), suggest that Hcy does not accumulate in E. coli cells in the presence of acetate.

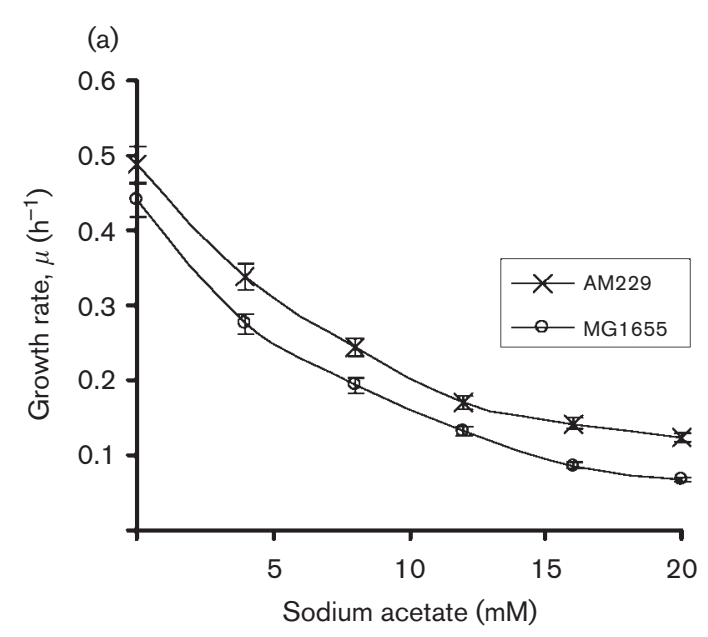

(b)

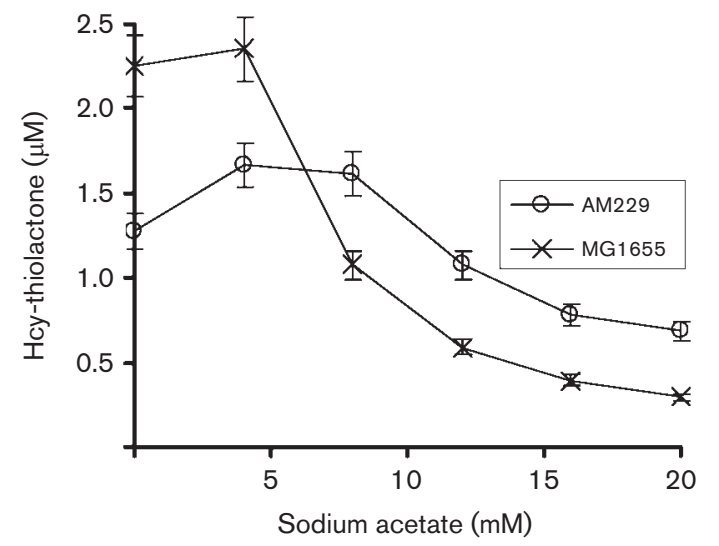

Fig. 4. The accumulation of Hcy-thiolactone does not increase in acetate-inhibited E. coli cultures. E. coli strains MG1655 and AM229 were grown in minimal medium supplemented with 0$20 \mathrm{mM}$ sodium acetate. Growth was monitored and Hcythiolactone assayed as described in Methods. Growth rates (a) and Hcy-thiolactone concentrations (b) are plotted as a function of sodium acetate concentration. 


\section{Ile, Leu or Met accelerate the growth and inhibit the accumulation of Hcy-thiolactone in Hcy- supplemented cultures: growth rate depends on Hcy-thiolactone synthesis}

The inhibition of E. coli growth by Hcy has been suggested to be caused by the inhibition of threonine deaminase by Hcy, which leads to isoleucine auxotrophy (Tuite et al., 2005). Consistent with this conclusion, the growth inhibition was reversed by supplementation of bacterial cultures with Ile, but not with Met or Leu (Tuite et al., 2005). However, the growth-stimulatory effect of Ile in Hcy-inhibited cultures could also be due to the prevention by Ile of the metabolic conversion of Hcy to Hcythiolactone catalysed by IleRS (Jakubowski \& Fersht, 1981; Jakubowski, 1995). In E. coli exogenous Hcy is metabolized to Hcy-thiolactone by MetRS, IleRS and LeuRS (Fig. 1). Supplementation of bacterial cultures with an amino acid cognate to MetRS (Met), IleRS (Ile) or LeuRS (Leu) is known to prevent Hcy-thiolactone synthesis specifically by that synthetase (Jakubowski, 1995). If the synthesis of Hcy-thiolactone is growth inhibitory, then preventing Hcy-thiolactone synthesis should accelerate growth. To test this prediction, we examined effects of supplementation with Ile, Met or Leu on the growth of Hcy-inhibited E. coli MG1655 cultures. We found that cultures supplemented with both Hcy and Ile grew almost as well as cultures without Hcy (Fig. 5a). Supplementation with Leu or Met also accelerated growth of Hcy-containing cultures (Fig. 5b, c), albeit to a lower extent than the supplementation with Ile. Control experiments demonstrated that Ile, Leu or Met did not accelerate bacterial growth in the absence of Hcy (not shown).

To examine the relationship between growth rate and Hcythiolactone, E. coli MG1655, PhB1907 and AM228 cultures were grown with a series of DL-Hcy concentrations in the absence and presence of Ile. Growth was monitored and Hcythiolactone measured. Growth rates and normalized Hcythiolactone concentrations were calculated. We found that in the absence of Ile, growth rate correlated with the normalized Hcy-thiolactone concentration (Hcy-thiolactone/growth rate) (Fig. 6a-c). In the presence of Ile, normalized Hcythiolactone concentrations were lowered and the growth accelerated at each DL-Hcy concentration tested; the experimental points shifted from the lower right to the upper left part of the graphs. Because Ile does not prevent Hcy transport into E. coli cells (Jakubowski, 1995; Tuite et al., 2005), these data suggest that the growth-stimulatory effect of Ile under these conditions is at least in part due to the inhibition of Hcy-thiolactone accumulation by Ile.

We then examined the effects of various concentrations of Ile, Met and Leu on growth rates and Hcy-thiolactone accumulation in Hcy-inhibited cultures. We found that normalized Hcy-thiolactone concentrations decreased whereas growth rates increased in the presence of increasing concentrations of Ile, Met or Leu in bacterial cultures containing a constant DL-Hcy concentration (Fig. 6d). (a)
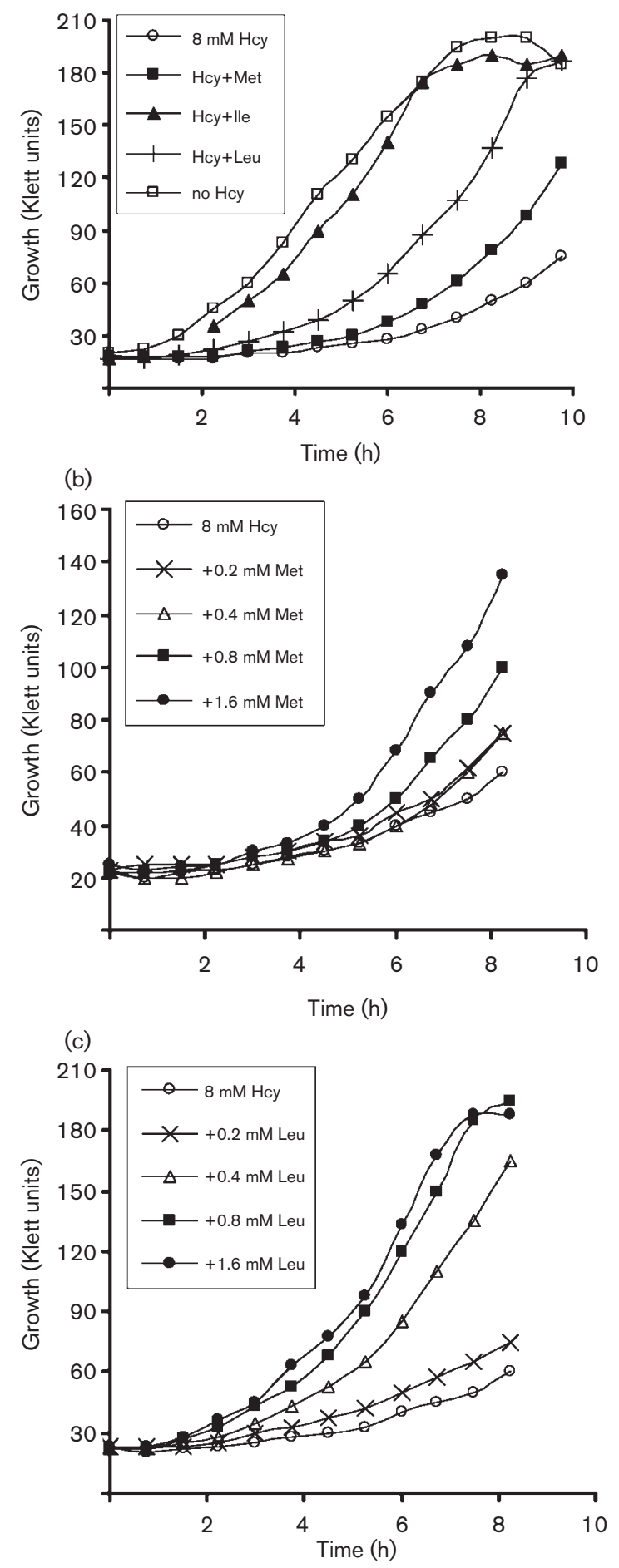

Fig. 5. Supplementation with lle, Leu or Met accelerates growth of Hcy-inhibited E. coli cultures. (a) E. coli strain MG1655 was grown in minimal medium supplemented with 0 or $8 \mathrm{mM}$ DL-Hcy and $0.4 \mathrm{mM}$ Met, lle or Leu. (b, c) E. coli strain MG1655 was grown in minimal medium supplemented with $8 \mathrm{mM}$ DL-Hcy and 0 , $0.2,0.40 .8$ and $1.6 \mathrm{mM}$ Met (b) or Leu (c). 100 Klett units correspond to $2 \times 10^{8}$ cells $\mathrm{ml}^{-1}$. 
(a) E. coli PhB1907

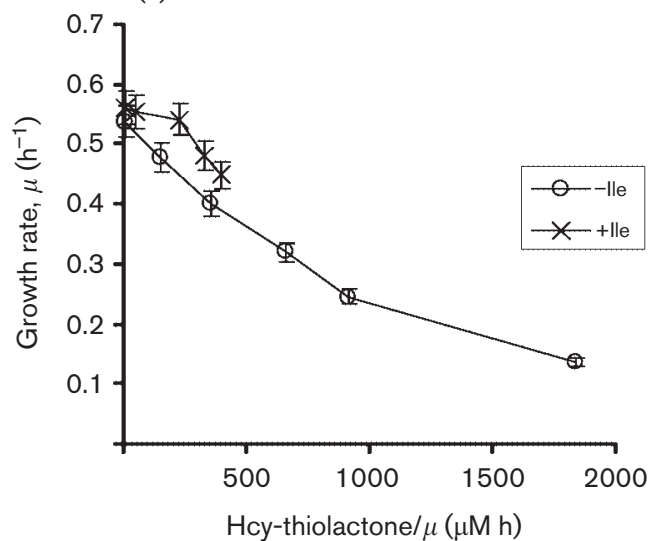

(c) E. coli MG1655

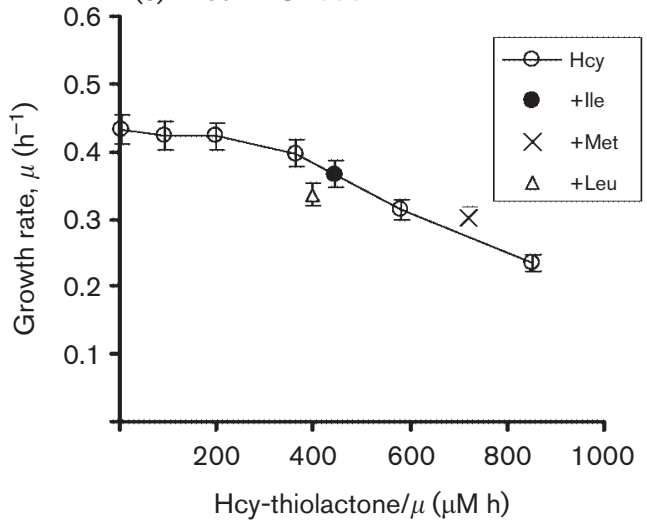

(b) E. coli AM228

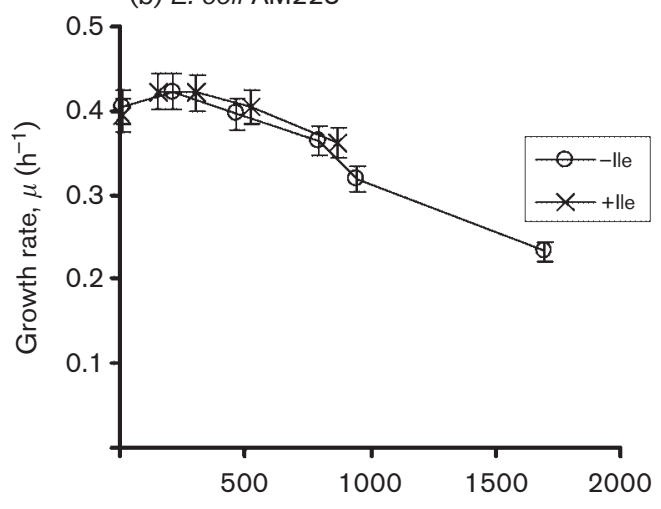

Hcy-thiolactone/ $\mu(\mu \mathrm{M} \mathrm{h})$

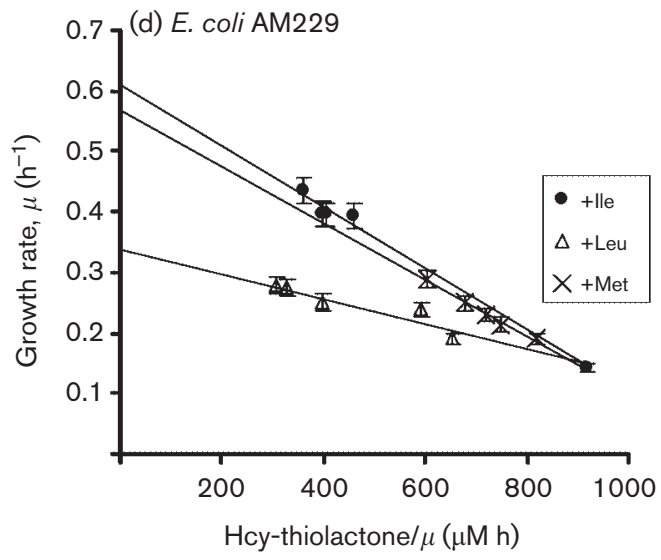

Fig. 6. Growth rate depends on Hcy-thiolactone accumulation. (a, b) E. coli strains PhB1907 (a) and AM228 (b) were grown in minimal medium supplemented with $0,0.5,1,2,4$ or $8 \mathrm{mM}$ DL-Hcy in the absence $(\bigcirc)$ and presence $(\times)$ of $1.6 \mathrm{mM}$ lle. (c) $E$. coli strain MG1655 was grown on minimal medium supplemented with 0, 0.5, 1, 2, 4 or $8 \mathrm{mM}$ DL-Hcy (o) or with $8 \mathrm{mM}$ DL$\mathrm{Hcy}+0.4 \mathrm{mM}$ Ile $(\bullet)$, Leu $(\triangle)$ or Met $(\times)$. (d) E. coli strain AM229 was grown in minimal medium supplemented with $8 \mathrm{mM}$ DLHcy and $0,0.2,0.4,0.8$ or $1.6 \mathrm{mM} \mathrm{lle}(\bullet)$, Leu $(\triangle)$ or Met $(\times)$. Growth rates were calculated and Hcy-thiolactone assayed at the end of growth experiments as described in Methods. Growth rates are plotted as a function of Hcy-thiolactone/growth rate.

Because Ile, Met or Leu do not prevent Hcy transport into $E$. coli cells (Jakubowski, 1995), and do not affect bacterial growth rate in the absence of Hcy (not shown), the growthstimulatory effect is most likely due to the inhibition of Hcythiolactone synthesis by these amino acids. Taken together, our results suggest that the increased synthesis of Hcythiolactone catalysed by IleRS, MetRS and LeuRS contributes to growth inhibition in Hcy-supplemented cultures.

\section{Hcy incorporation into protein}

The finding that the growth rate is affected by the accumulation of Hcy-thiolactone raises the question whether the synthesis of Hcy-thiolactone (Fig. 1) is growth inhibitory per se or whether subsequent protein $\mathrm{N}$ homocysteinylation by Hcy-thiolactone (Fig. 2) is causing growth inhibition. To determine whether $\mathrm{N}$-linked Hcy accumulates in E. coli proteins we used a new sensitive HPLC-based assay (Jakubowski, 2008). To examine possible involvement of specific proteases in its turnover, we studied effects of deletion of Lon or Clp proteases on the accumulation of protein $\mathrm{N}$-linked $\mathrm{Hcy}$.

We found that the level of protein $N$-linked Hcy in E. coli strain MG1655 grown in a minimal medium in the absence of exogenous Hcy was $58 \pm 17$ pmol per $2 \times 10^{8}$ cells (Table 1). Protease-deficient strains, AM228 ( $\Delta$ lon) and PhB1907 $(\triangle c l p X P)$, accumulated similar levels of protein $N$-linked Hcy: $43 \pm 13$ pmol and $73 \pm 26$ pmol per $2 \times 10^{8}$ cells, respectively (Table 1 ).

In E. coli MG1655 cultures supplemented with $4 \mathrm{mM}$ DL$\mathrm{Hcy}$, the levels of protein $\mathrm{N}$-linked $\mathrm{Hcy}$ increased significantly, from 58 pmol per $2 \times 10^{8}$ cells in cultures maintained in the absence of Hcy, to 151 pmol per $2 \times 10^{8}$ cells at $0.5 \mathrm{~h}$ and $200 \mathrm{pmol}$ per $2 \times 10^{8}$ cells at $4 \mathrm{~h}$ in the presence of Hcy (Table 1). In the presence of Hcy for 0.5$4 \mathrm{~h}$ the AM228 $(\Delta l o n)$ and PhB1907 $(\Delta c l p X P)$ cultures accumulated 106-315 pmol protein $N$-linked Hcy per 
Table 1. Levels of $N$-linked Hcy in E. coli protein

The values tabulated are means $\pm \mathrm{SD}$ from two independent experiments.

\begin{tabular}{|c|c|c|c|}
\hline \multirow{2}{*}{$\begin{array}{l}\text { Time (h) } \\
\text { with } 4 \mathrm{mM} \\
\text { DL-Hcy }\end{array}$} & \multicolumn{3}{|c|}{$\begin{array}{c}\text { Protein } N \text {-linked Hcy } \\
\left(\text { pmol per } 2 \times 10^{8} \text { cells) in } E \text {. coli strain }\right.\end{array}$} \\
\hline & MG1655 & AM228 & PhB1907 \\
\hline 0 & $58 \pm 17^{\star}$ & $43 \pm 13^{*}$ & $73 \pm 26$ \\
\hline 0.5 & 151 & 106 & 238 \\
\hline 1 & & 150 & 149 \\
\hline 2 & $301 \pm 62^{*}$ & $223 \pm 3^{*}$ & $142 \pm 68$ \\
\hline 4 & 200 & $315 \pm 169$ & $209 \pm 90$ \\
\hline 24 & $337 \pm 26^{*} \dagger$ & $703 \pm 11^{*} \dagger$ & $641 \pm 273$ \\
\hline
\end{tabular}

${ }^{*}$ For strains MG1655 and AM228 the differences between $0 \mathrm{~h}$ and $2 \mathrm{~h}$ or $24 \mathrm{~h}$ cultures are statistically significant ( $P=0.04$ to 0.001 ).

$\dagger$ The differences between $24 \mathrm{~h}$ MG1655 and AM228 cultures are statistically significant $(P=0.01)$.

$2 \times 10^{8}$ cells and 238-209 pmol protein $N$-linked Hcy per $2 \times 10^{8}$ cells, respectively, similar to the levels of protein $N$ linked Hcy accumulated by the wild-type E. coli strain MG1655. There were further increases in the accumulation of protein $\mathrm{N}$-linked Hcy after $24 \mathrm{~h}$ incubation with Hcy, but only the increase observed in strain AM228 $(\Delta l o n)$ was statistically significant $(P=0.01)$. Taken together, these findings suggest that neither Lon nor Clp protease alone contributes significantly to $\mathrm{N}$-Hcy-protein turnover in $E$. coli under short-term growth conditions. However, the Lon protease may participate in $\mathrm{N}$-Hcy-protein turnover under long-term stationary-phase conditions.

It should be noted that under the same conditions Hcythiolactone accumulates at a rate of 470 and $44300 \mathrm{pmol} \mathrm{h}^{-1}$ per ml culture, reaching the levels of 1900 and 177300 pmol per ml culture after $4 \mathrm{~h}$ in the absence and presence of exogenous $\mathrm{Hcy}$, respectively. Thus, protein $\mathrm{N}$ linked Hcy represents $3 \%$ or $0.14 \%$ of its precursor Hcythiolactone, in the absence or presence of exogenous Hcy, respectively. Relatively small ( $\sim 4$-fold on average) increases in protein $\mathrm{N}$-linked Hcy during $4 \mathrm{~h}$ compared with a large $(\sim 100$-fold) increase in Hcy-thiolactone accumulation observed in Hcy-supplemented cultures suggests that Hcythiolactone-mediated incorporation of Hcy into protein is not likely to contribute significantly to the growth inhibition observed in the presence of Hcy. This conclusion is also supported by the observation that supplementation with $0.5 \mathrm{mM}$ Hcy-thiolactone does not inhibit the growth of $E$. coli (Tuite et al., 2005).

\section{Energy costs of Hcy-thiolactone synthesis in Hcy- supplemented cultures}

Protein synthesis consumes $95 \%$ of the energy needed for macromolecule biosynthesis in E. coli (Ingraham et al.,
1983). Taking that one molecule of ATP and two molecules of GTP are required for incorporation of each amino acid into protein (=four high-energy bonds, two for $\mathrm{ATP} \rightarrow \mathrm{AMP}$ during aminoacyl-tRNA synthesis and two for $2 \mathrm{GTP} \rightarrow 2 \mathrm{GDP}$ during subsequent ribosomal protein synthesis) it has been estimated that $\sim 2 \%$ of the total energy required for protein synthesis in E. coli is expended for editing under balanced growth conditions (Ingraham et al., 1983).

Bacterial ATP production is estimated from substrate consumption and generation of metabolites such as lactic acid and acetate (Teusink et al., 2005). In an analogous way we can estimate ATP consumption from a metabolite such as Hcy-thiolactone (Fig. 1). In E. coli growing in a minimal medium one molecule of Hcy is edited by the conversion to Hcy-thiolactone per 109 molecules of Met incorporated into protein (Jakubowski, 1990; Jakubowski \& Goldman, 1992; Jakubowski, 1993). Because one mole of ATP is hydrolysed per mole of Hcy-thiolactone formed (Fig. 1) (Jakubowski, 1997b) and Met activation also consumes one mole of ATP per mole of Met-tRNA formed (Mulvey \& Fersht, 1977), under these conditions unproductive ATP hydrolysis associated with Hcy editing is relatively low, equal to $\sim 1 \%$ of productive ATP consumption for Met activation for protein synthesis. However, the accumulation of Hcy-thiolactone increases 180-, 45-, 130- and 150fold (from 1.0, 5.0, 2.4 and $1.4 \mu \mathrm{M}$ in the absence to 180, 220, 300 and $210 \mu \mathrm{M}$ in the presence of $4 \mathrm{mM} \mathrm{Hcy}$ ) in cultures of E. coli strains MG1655, PhB1907, AM228 and AM229, respectively (Fig. 3). This means that in the presence of exogenous Hcy on average one mole of ATP is unproductively dissipated for Hcy-thiolactone synthesis per mole of ATP that is consumed for Met activation, i.e. unproductive ATP dissipation is equal to $100 \%$ of productive ATP consumption. As Met constitutes 2.9\% of the total amino acid content in bacterial protein (Ingraham et al., 1983), one can estimate that ATP energy dissipation for Hcy editing increases from $0.029 \%$ in minimal medium to $2.9 \%$ in Hcy-supplemented cultures, relative to the total energy required for protein synthesis in E. coli. Given the accuracy of available analytical methods, this level of extra ATP consumption for Hcy editing would be difficult to measure experimentally. However, given that ATP production is related to the growth rate (Teusink et al., 2005), the increased energy dissipation could contribute to the growth inhibition observed in Hcy-supplemented E. coli cultures. This conclusion is supported by our findings that in Hcy-supplemented cultures the inhibition of Hcy-thiolactone accumulation accelerates growth (Fig. 5 ) and the growth rate is inversely related to Hcythiolactone accumulation (Fig. 6). Furthermore, this conclusion is consistent with previous findings in yeast showing that a strain in which unproductive energy dissipation for Hcy editing represents just $13 \%$ of the energy used for activation of Met grows more slowly than an isogenic strain which dissipates only $0.95 \%$ energy (Jakubowski, 1991). 
Hcy could potentially affect bacterial growth by inhibiting the Met-tRNA synthetase activity of MetRS. The extent of the inhibition can be estimated from our finding that unproductive ATP dissipation for Hcy editing is equal to $100 \%$ of productive ATP consumption for Met activation in Hcy-inhibited cultures. Thus, under these conditions $50 \%$ of MetRS molecules in the bacterial cell are engaged in Met-tRNA synthesis whereas the other $50 \%$ are engaged in Hcy-thiolactone synthesis, i.e. the inhibition of MettRNA synthetase activity by Hcy is $50 \%$. However, because cellular levels of MetRS are not rate-limiting for protein synthesis (Jakubowski, 1990; Jakubowski \& Goldman, 1992), this level of MetRS inhibition is unlikely to affect bacterial growth.

\section{Effects of Hcy on cell viability}

We then examined effects of Hcy on cell viability in cultures of E. coli strains MG1655, AM228 ( $\Delta l o n)$ and PhB1907 $(\Delta c l p)$. We found that exposure of E. coli cultures to $4 \mathrm{mM} \mathrm{Hcy}$ at $37{ }^{\circ} \mathrm{C}$ for up to $72 \mathrm{~h}$ diminished cell viability in a time-dependent manner (Fig. 7). Strain MG1655 was more sensitive to Hcy than the proteasedeficient strains AM228 ( $\Delta$ lon $)$ and PhB1907 $(\Delta c l p X P)$. The exposure to Hcy did not affect the induction of heatshock proteins in any of the strains examined (not shown). We also found that supplementation of Hcy-containing cultures with Ile, Leu or Met (1.6 mM each) did not prevent the loss of cell viability caused by Hcy (not shown). These findings suggest that Hcy-thiolactone accumulation (Fig. 4) or Ile starvation (Tuite et al., 2005) are unlikely to cause cell death under these experimental conditions. Furthermore, the modest increases in $\mathrm{N}$-Hcy-protein levels (Table 1) suggest that $\mathrm{N}$-Hcy-protein accumulation is unlikely to be responsible for the loss of cell viability in E. coli.

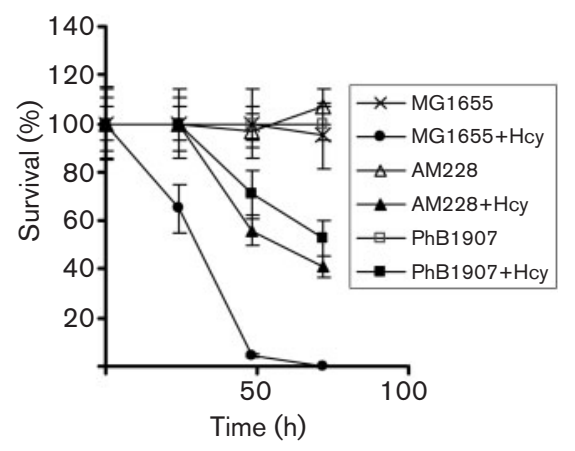

Fig. 7. Hcy affects long-term survival of $E$. coli cultures. E. coli strains MG1655, PhB1907 and AM228 were grown in minimal medium supplemented or not with $4 \mathrm{mM}$ DL-Hcy. After 0, 1, 2 and 3 days at $37{ }^{\circ} \mathrm{C}$ aliquots of cultures were diluted and plated on LB-agar for colony counts. Percentage surviving cells is plotted vs time. Each point is the mean of three independent determinations. Standard deviations are indicated by error bars.

\section{Conclusions}

Our data show the following: (1) growth inhibition caused by supplementation of $E$. coli cultures with $\mathrm{Hcy}$ is accompanied by greatly increased synthesis of Hcythiolactone, which requires increased unproductive ATP dissipation; (2) inhibition of the synthesis of Hcythiolactone accelerates bacterial growth; (3) growth rates are inversely related to the accumulation of Hcy-thiolactone; (4) supplementation with Hcy modestly increases the levels of protein $N$-linked Hcy in E. coli cells. Taken together, these results suggest that Hcy editing contributes to growth inhibition in E. coli.

\section{ACKNOWLEDGEMENTS}

We thank Zafri Humayun and Abu Amar Al Mamun for providing E. coli strains. This work was supported by grants from the American Heart Association (0855919D) and the Ministry of Science and Higher Education, Poland (N401 065 32/1504 and N401 132 32/ 2670).

\section{REFERENCES}

Chwatko, G. \& Jakubowski, H. (2005). The determination of homocysteine-thiolactone in human plasma. Anal Biochem 337, 271-277.

Chwatko, G., Boers, G. H., Strauss, K. A., Shih, D. M. \& Jakubowski, H. (2007). Mutations in methylenetetrahydrofolate reductase or cystathionine beta-synthase gene, or a high-methionine diet, increase homocysteine thiolactone levels in humans and mice. FASEB J 21, 1707-1713.

Gao, W., Goldman, E. \& Jakubowski, H. (1994). Role of carboxyterminal region in proofreading function of methionyl-tRNA synthetase in Escherichia coli. Biochemistry 33, 11528-11535.

Ingraham, J. L., Maaløe, O. \& Neidhardt, F. C. (1983). Growth of the Bacterial Cell. Sunderland, MA: Sinauer Associates.

Jakubowski, H. (1990). Proofreading in vivo: editing of homocysteine by methionyl-tRNA synthetase in Escherichia coli. Proc Natl Acad Sci U S A 87, 4504-4508.

Jakubowski, H. (1991). Proofreading in vivo: editing of homocysteine by methionyl-tRNA synthetase in the yeast Saccharomyces cerevisiae. EMBO J 10, 593-598.

Jakubowski, H. (1993). Energy cost of proofreading in vivo: the charging of methionine tRNAs in Escherichia coli. FASEB J 7, 168-172.

Jakubowski, H. (1995). Proofreading in vivo. Editing of homocysteine by aminoacyl-tRNA synthetases in Escherichia coli. J Biol Chem 270, 17672-17673.

Jakubowski, H. (1997a). Metabolism of homocysteine thiolactone in human cell cultures. Possible mechanism for pathological consequences of elevated homocysteine levels. J Biol Chem 272, 1935-1942.

Jakubowski, H. (1997b). Aminoacyl thioester chemistry of class II aminoacyl-tRNA synthetases. Biochemistry 36, 11077-11085.

Jakubowski, H. (1999a). Misacylation of tRNALys with noncognate amino acids by lysyl-tRNA synthetase. Biochemistry 38, 8088-8093.

Jakubowski, H. (1999b). Protein homocysteinylation: possible mechanism underlying pathological consequences of elevated homocysteine levels. FASEB J 13, 2277-2283.

Jakubowski, H. (2000). Translational incorporation of S-nitrosohomocysteine into protein. J Biol Chem 275, 21813-21816. 
Jakubowski, H. (2001a). Protein $N$-homocysteinylation: implications for atherosclerosis. Biomed Pharmacother 55, 443-447.

Jakubowski, H. (2001b). Translational accuracy of aminoacyl-tRNA synthetases: implications for atherosclerosis. J Nutr 131, 2983S-2987S.

Jakubowski, H. (2002a). The determination of homocysteinethiolactone in biological samples. Anal Biochem 308, 112-119.

Jakubowski, H. (2002b). Homocysteine is a protein amino acid in humans. Implications for homocysteine-linked disease. J Biol Chem 277, 30425-30428.

Jakubowski, H. (2004). Molecular basis of homocysteine toxicity in humans. Cell Mol Life Sci 61, 470-487.

Jakubowski, H. (2005a). tRNA synthetase editing of amino acids. In Encyclopedia of Life Sciences http://mrw.interscience.wiley.com/emrw/ 9780470015902/els/article/a0000532/current/abstract doi:10.1038/npg. els.0003933.

Jakubowski, H. (2005b). Accuracy of aminoacyl-tRNA synthetases: proofreading of amino acids. In The Aminoacyl-tRNA Synthetases, pp. 384-396. Edited by M. Ibba, C. Francklyn \& S. Cusack. Georgetown, TX: Landes Bioscience/Eurekah.com.

Jakubowski, H. (2006a). Pathophysiological consequences of homocysteine excess. J Nutr 136, 1741S-1749S.

Jakubowski, H. (2006b). Mechanism of the condensation of homocysteine thiolactone with aldehydes. Chemistry 12, 8039-8043.

Jakubowski, H. (2007a). The molecular basis of homocysteine thiolactone-mediated vascular disease. Clin Chem Lab Med 45, 1704-1716.

Jakubowski, H. (2007b). Facile syntheses of $\left[{ }^{35} S\right]$ homocysteinethiolactone, $\left[{ }^{35}\right.$ S]homocystine, $\left[{ }^{35}\right.$ S $]$ homocysteine, and $\left[S\right.$-nitroso- $\left.{ }^{35} \mathrm{~S}\right]$ homocysteine. Anal Biochem 370, 124-126.

Jakubowski, H. (2008). New method for the determination of protein $N$-linked homocysteine. Anal Biochem 380, 257-261.
Jakubowski, H. \& Fersht, A. R. (1981). Alternative pathways for editing non-cognate amino acids by aminoacyl-tRNA synthetases. Nucleic Acids Res 9, 3105-3117.

Jakubowski, H. \& Goldman, E. (1992). Editing of errors in selection of amino acids for protein synthesis. Microbiol Rev 56, 412-429.

Karkhanis, V. A., Mascarenhas, A. P. \& Martinis, S. A. (2007). Amino acid toxicities of Escherichia coli that are prevented by leucyl-tRNA synthetase amino acid editing. J Bacteriol 189, 8765-8768.

Kumar, A., John, L., Alam, M. M., Gupta, A., Sharma, G., Pillai, B. \& Sengupta, S. (2006). Homocysteine- and cysteine-mediated growth defect is not associated with induction of oxidative stress response genes in yeast. Biochem J 396, 61-69.

Mulvey, R. S. \& Fersht, A. R. (1977). Editing mechanisms in aminoacylation of tRNA: ATP consumption and the binding of aminoacyl-tRNA by elongation factor Tu. Biochemistry 16, 47314737.

Roe, A. J., O'Byrne, C., McLaggan, D. \& Booth, I. R. (2002). Inhibition of Escherichia coli growth by acetic acid: a problem with methionine biosynthesis and homocysteine toxicity. Microbiology 148, 2215-2222.

Teusink, B., van Enckevort, F. H., Francke, C., Wiersma, A., Wegkamp, A., Smid, E. J. \& Siezen, R. J. (2005). In silico reconstruction of the metabolic pathways of Lactobacillus plantarum: comparing predictions of nutrient requirements with those from growth experiments. Appl Environ Microbiol 71, 7253-7262.

Tuite, N. L., Fraser, K. R. \& O'Byrne, C. P. (2005). Homocysteine toxicity in Escherichia coli is caused by a perturbation of branchedchain amino acid biosynthesis. J Bacteriol 187, 4362-4371.

Zimny, J., Sikora, M., Guranowski, A. \& Jakubowski, H. (2006). Protective mechanisms against homocysteine toxicity: the role of bleomycin hydrolase. J Biol Chem 281, 22485-22492.

Edited by: G. H. Thomas 